Nigerian Journal of Technology (NIJOTECH)

Vol. 38, No. 1, January 2019, pp. 1 - 7

Copyright@ Faculty of Engineering, University of Nigeria, Nsukka,

Print ISSN: 0331-8443, Electronic ISSN: 2467-8821

www.nijotech.com

http://dx.doi.org/10.4314/njt.v38i1.1

\title{
EFFECT OF LOW DENSITY POLYETHYLENE AS BITUMEN MODIFIER ON SOME PROPERTIES OF HOT MIX ASPHALT
}

\author{
D. B. Eme ${ }^{1, *}$ and C. Nwaobakata ${ }^{2}$ \\ 1, 2, Department of Civil Engineering, University of Port HarCourt, CHOBA, Rivers State, Nigeria \\ E-mail addresses: ${ }^{1}$ dennis.eme@uniport.edu.ng, ${ }^{2}$ makus20002000@gmail.com
}

\begin{abstract}
In Nigeria today, polyethylene or water sachet is a major environmental pollutant, a nonbiodegradable material. The usefulness of this pollutant (polyethylene) in the highway industry was investigated by studying its effects on some selected properties of Hot Mix Asphalt, such as, bulk density, stability and flow of the asphalt concrete mix. Specimen preparation was done using Marshall Mix design procedure. The optimum binder content was determined as $5.20 \%$ and three samples each for five variations of polyethylene content (2\%, 4\%, 6\%, $8 \%$ and $10 \%)$ by weight of optimum binder content. It was observed from the study, that the stability and density of asphalt increased with polyethylene content, while a linear reduction in the flow and penetration values was observed with polyethylene content. The optimum modifier content value of $8 \%$ by weight of the optimum bitumen content. Models were also developed whose predictive values corroborated well with experimental values with acceptable coefficient of correlation values. Bitumen modified with polyethylene improved the engineering properties of asphalt which therefore means that usage of this waste product in the asphalt production for roadwork is an effective and economical way of managing this waste.
\end{abstract}

Keywords: Polyethylene, modifier, asphalt, bitumen

\section{INTRODUCTION}

Asphalt is a composite material commonly used to surface roads, parking lots, airports, as well as the core of embankment dams. It is also known as blacktop or pavement in North America and tarmac or bitumen macadam in the United Kingdom and Ireland [1].

Asphalt consists basically of three elements; aggregates, binder and filler. The aggregates are bounded together with the binder which is called bitumen, which serves the same purpose as cement in concrete.

The quality of the bitumen, which is an essential component of asphalt, is very vital as it goes a long way to determine the strength and durability of the asphalt. Improvement of the quality of bitumen has been encouraged over the years keeping in mind the financial implications. The use of virgin polymers in bitumen to improve the characteristics of resulting polymer modified bitumen has been accomplished for many years. Recently, there is interest in the substitution of commercial virgin material with recycled polymers. Author of [2] pointed out that when recycled polymers are used as bitumen modifying agents, the resulting mixture may show similar performance to those containing virgin polymer.

Bitumen modification is not a new phenomenon. As early as 1923, natural and synthetic polymer modifications of bitumen have been patented [3, 4]. During the 1930's, test projects were constructed in Europe [4]. In the mid, 1980s Australia started using polymers in bituminous mixtures, which is evident from the current national asphalt specifications. In [5] it was suggested that the use of rubberized bitumen in road construction in Malaysia. In [6], it was stated that bituminous materials obtained from different sources contain different proportions of different constituents and hence different properties. To judge their suitability as binders, tests such as

* Corresponding author, tel: +234 $703-800-9751$ 
flash point, softening and penetration tests need to be carried out.

Polyethylene has been found to be one of the most effective polymer additives. Polyethylene is the most popular plastic in the world [7]. Plastic is a versatile material. Due to the industrial revolution, and its large scale production, plastic seemed to be a cheaper and effective raw material. Today every vital sector of the economy starting from agriculture to packaging automobile, electronics, electrical, building construction sectors have been virtually revolutionized by the application of plastics. Several studies have proven the health hazard caused by improper disposal of plastic wastes such as reproductive; problems in humans and animals, genital abnormalities etc. [8]. Looking forward into the scenario of present life style, a complete ban on plastic usage cannot be considered but these plastic wastes can be reused. Thus, the purpose of this study is to investigate the possibility of using the waste polyethylene material (pure water sachet) as a bitumen modifier. The study will compare the physical and rheological properties of the modified asphalt concrete to conventional or unmodified asphalt.

\section{MATERIAL AND METHOD}

\subsection{Materials}

The bitumen used for this study was obtained from a material shop in Port Harcourt, Nigeria.

The coarse aggregate used was crushed angular granite obtained from a construction site in Port Harcourt, Nigeria.

The filler used for the experiment was that portion of fine aggregate finer than sieve $0.212 \mathrm{~mm}$ sourced from the same location as the coarse aggregate.

Light density polymer used for the experiment are waste sachet water bags sourced from the University of Port Harcourt, Environment.

\subsection{Experimental Equipment}

The equipment that were used for the purpose of this study are outlined below:

a. The Ring and Ball apparatus used for the softening point test.

b. The Standard Penetrometer apparatus used for the penetration test.

c. Marshall Mix Design apparatus.

d. Other apparatus include, set of sieves, oven, hand brush, pan, scoop, sensitive weighing balance with an accuracy of $0.01 \mathrm{~g}$

\section{METHODS}

\subsection{Experimental Mix Design}

The sourced bitumen and aggregates were subjected to preliminary tests like the softening point test, penetration tests and gradation tests to ensure that these materials meets the a minimum specifications. The whole experimental set up was designed for the medium traffic roads with properties outlined in Table 1.

Table 1: Properties of Asphalt concrete in medium traffic category

\begin{tabular}{lc}
\hline Mix criteria & Medium traffic \\
\hline No. of blow on each face & 50 \\
Stability (minimum) & $3336-7566 \mathrm{~N}$ \\
Flow & $2-4.5$ \\
Percentage air voids & $3-5$ \\
\hline
\end{tabular}

The experimental methodology of the study involved laboratory tests (Marshall, stability and flow test, softening point test) on three (3) specimens per set per experiment. The bitumen at $(4 \%, 4.5 \%, 5 \%$, $5.5 \%$ and $6 \%$ ) were combined with the graded aggregates and the optimum bitumen content was determined using Marshall test procedure to be $5.20 \%$. This optimum binder content was systematically, and partially replaced with properly prepared polyethylene bags at $2 \%, 4 \%, 6 \%, 8 \%$ and $10 \%$ by weight of the optimum bitumen content. In summary, the experimental mix design entails all the ratio and proportioning of materials required for the optimum result of the study. This study encompasses, the aggregate blending and proportioning and optimum binder content determination.

\subsubsection{Aggregates Proportion Blend}

For optimal mix design, the aggregates $A$ and $B$ were properly combined to meet gradation specifications. The aggregates were combined adopting the straight line approach and the optimal combination was obtained after the third trial to be $60 \%$ A and $40 \%$ B. This is shown as represented in Table 2 and Figure 1.

\subsubsection{Optimum Bitumen Content Determination}

In determining the Optimum Bitumen Content (OBC), the Marshall mix design procedure as specified in [9] was employed with a series of five (5) test sample with three (3) samples per experiment for $4 \%, 4.5 \%$, 
$5 \%, 5.5 \%$ and $6 \%$ bitumen content. The tests involved were, stability and flow test, bulk density test, determination of $\%$ air voids and determination of $\%$ voids in mineral aggregates. Each test sample weighed $1200 \mathrm{~g}$. The result of mix design used to determine $\mathrm{OBC}$ is presented in Figure $2(\mathrm{a}-\mathrm{e})$

Table 2: Optimum Trial Combination

\begin{tabular}{llllllll}
\hline Sieve size $(\mathrm{mm})$ & 40 & 20 & 10 & 4.75 & 2.36 & 0.6000 & 0.075 \\
\hline $\mathrm{A} * 0.60$ & 60 & 58.20 & 40.20 & 10.20 & 7.20 & 3.60 & 0.60 \\
$\mathrm{~B} * 0.40$ & 40 & 40 & 39.60 & 39.20 & 37.20 & 22.40 & 0.40 \\
Total & 100 & 98.20 & 79.80 & 49.40 & 44.40 & 26.0 & 1.0 \\
Specification & 100 & $80-100$ & $55-80$ & $40-60$ & $30-60$ & $15-30$ & $0-10$ \\
\hline
\end{tabular}

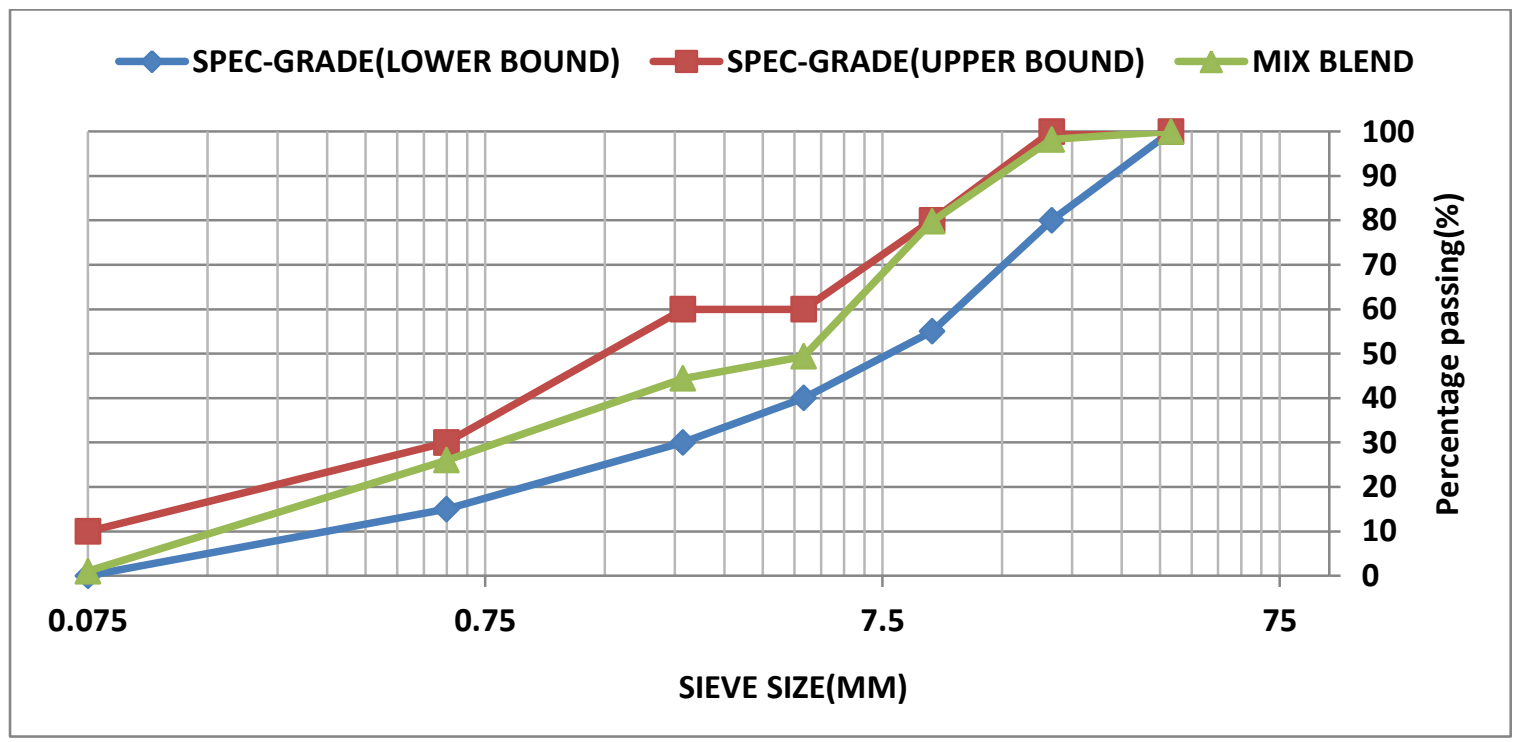

Figure 1: Aggregate gradation curve

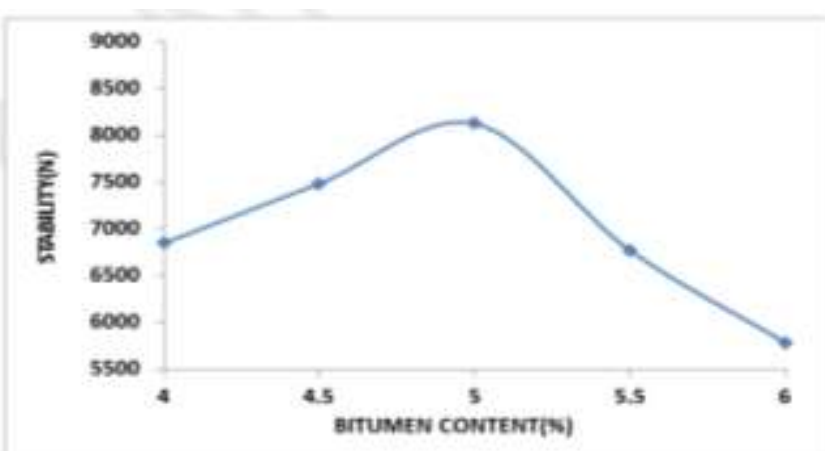

Figure 2a: Stability Values vs Percentage Bitumen Content

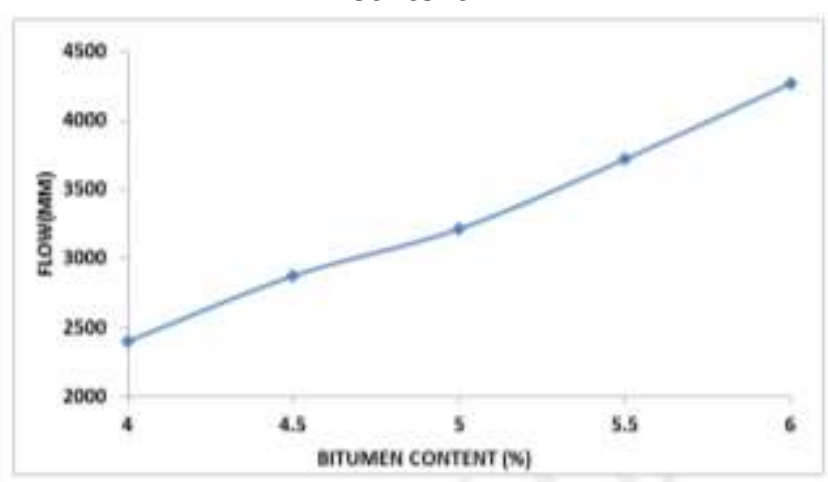

Figure 2b: Flow values vs percentage bitumen content

Nigerian Journal of Technology

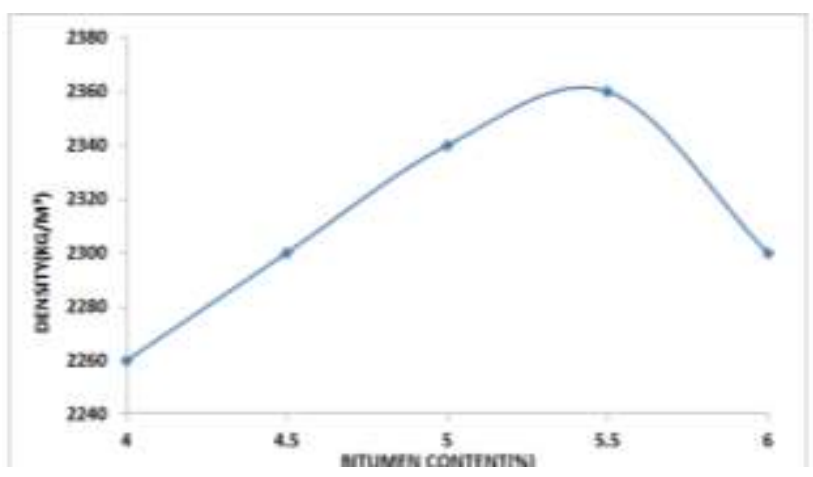

Figure 2c: Density Values Vs Percentage Bitumen Content

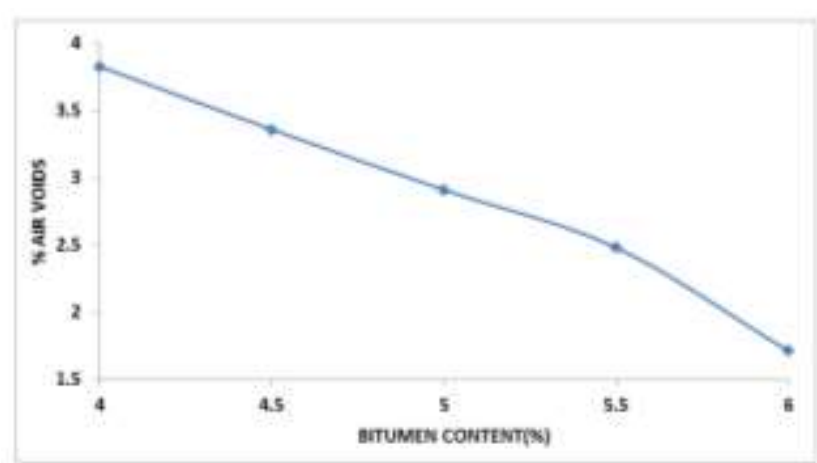

Figure 2d: Percent Air Void Vs Percent Bitumen Content

Vol. 38, No. 1, January, 2019 


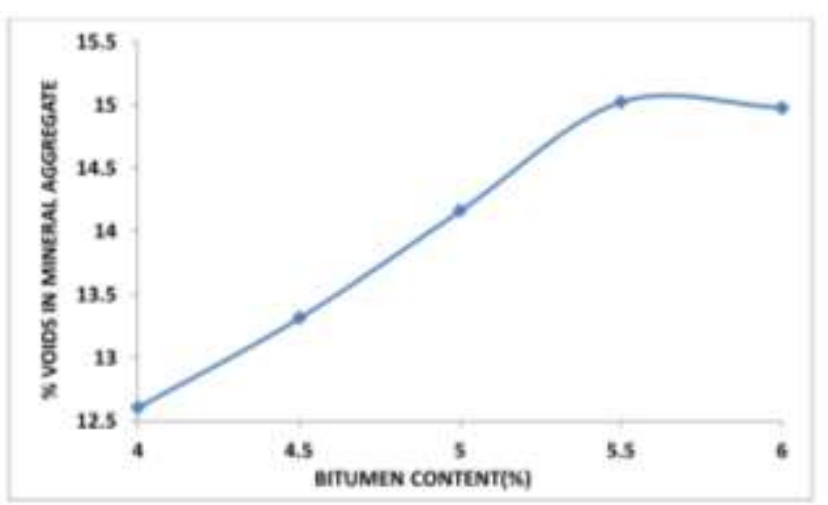

Figure 2e:: VMA VS Percent Bitumen Content

The OBC was thus obtained as $5.20 \%$ from the plots in Figure 2 adopting Equation 1.

$$
\mathrm{OBC}=\frac{A \cdot+B+C}{3}
$$

Where: $A$ is the Bitumen content at maximum stability, B is the Bitumen content at maximum density and $\mathrm{C}$ is the Bitumen content at medium air voids.

\subsection{Experimental Sample Preparation}

Bitumen without debris and adulterants was collected and heated to a temperature of above $100^{\circ} \mathrm{C}$ to transform it to liquid. The polyethylene was weighed with respect to $0 \%, 2 \%, 4 \%, 6 \%, 8 \%$ and $10 \%$ weight of the bitumen optimum bitumen content.
The weight of the pure liquid bitumen was measured into a steel cylinder and heated till it fully liquefied and was in a state to dissolve the light density polyethylene. The L.D.P bags were separately heated to liquefy before it was weighed and blended into measured bitumen and after continuous stirring by steel spoon, it was thoroughly mixed with hot bitumen.

The aggregate blend, was also weighed and heated in order to remove moisture as well as making the sample a Hot Mix Asphalt Concrete.

Table 4 shows the mix ratio for modified asphalt concrete

\section{RESULTS AND DISCUSSION}

\subsection{Results of Experimental Test}

The results of the Marshall stability and flow tests, specific gravity tests, softening point tests and penetration tests are clearly illustrated by Tables and Figures in this section.

\subsubsection{Marshal Stability and Flow Tests Result of Asphalt modified with LDP}

Figure 3 shows the result of the Marshall stability and flow tests for the modified bitumen asphalt.

Table 4: Mix Ratio for Modified Asphalt Concrete

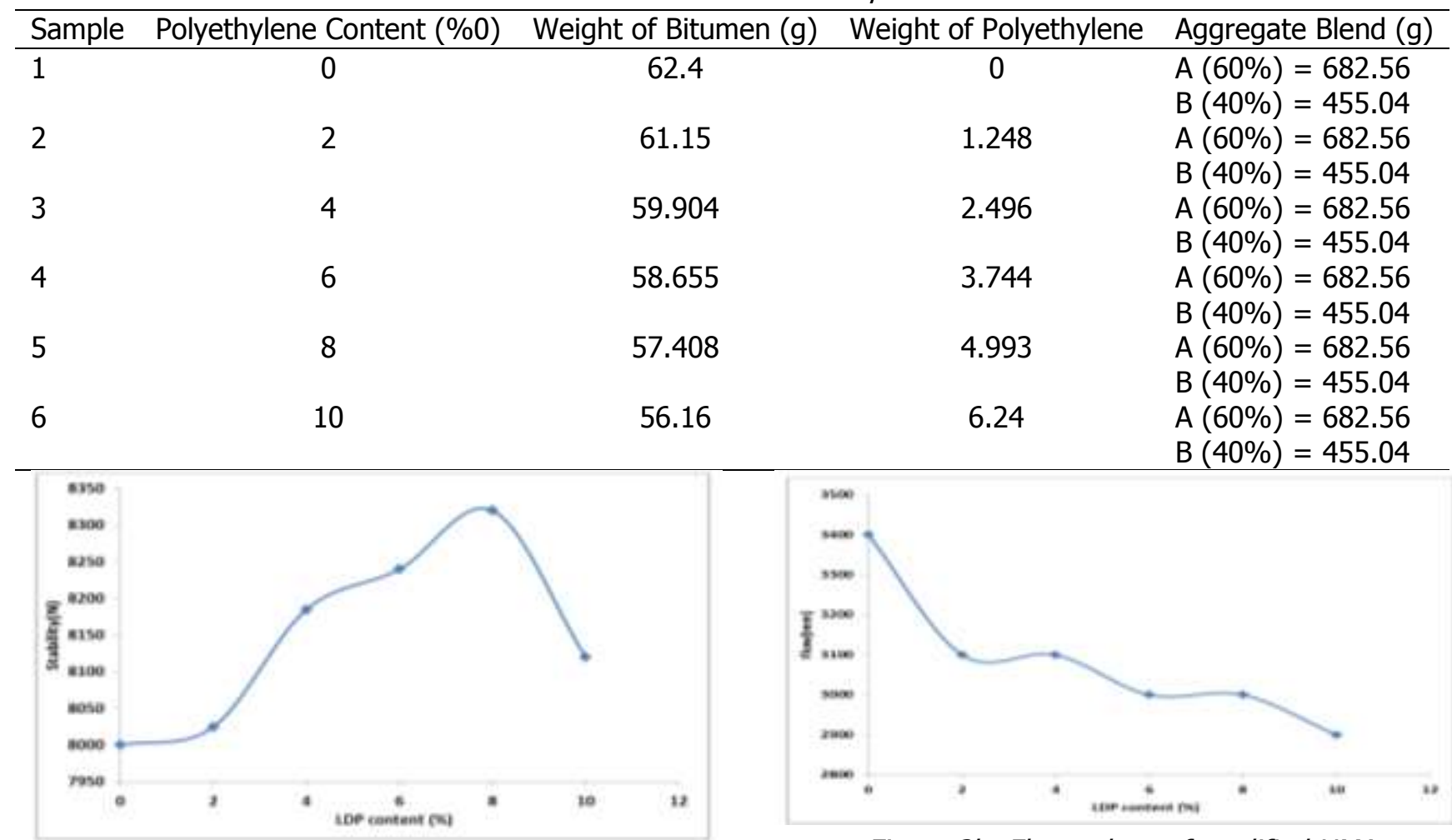

Figure 3a: Stability values of modified HMA

Figure 3b: Flow values of modified HMA 


\subsubsection{Specific Gravity Tests Result of Asphalt modified with LDP}

Figure 4 shows the result of specific gravity tests for the modified sample of asphalt.

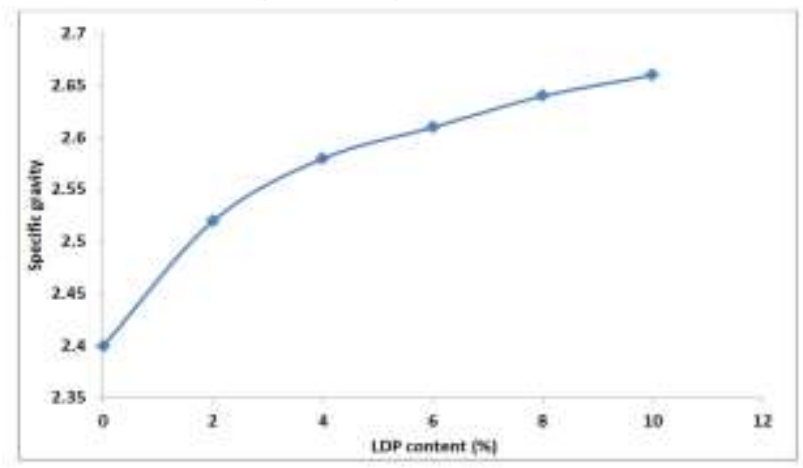

Figure 4: Specific gravity values of modified HMA

\subsubsection{Softening Point Tests Results of Asphalt modified with $L D P$}

The softening point test results for the modified asphalt are presented in Figure 5.

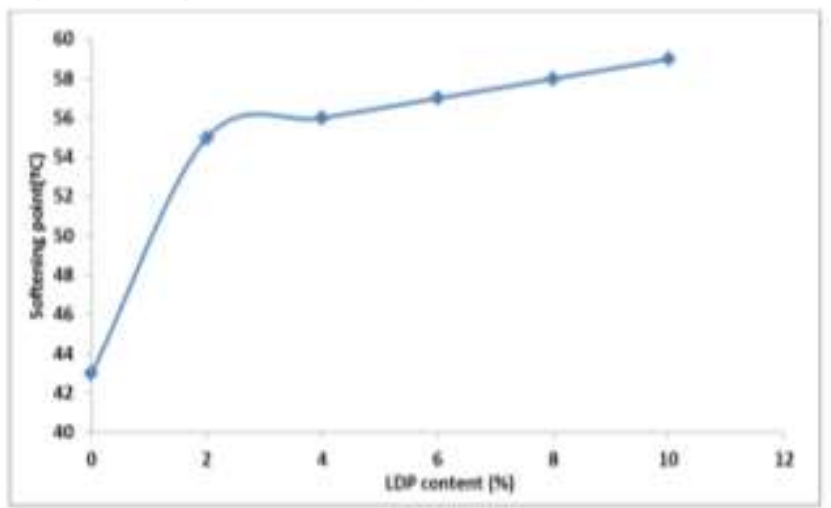

Figure 5: Softening point values of modified HMA

\subsubsection{Penetration Tests Results of Asphalt modified with LDP}

The penetration test result for the modified asphalt is presented in Figure 6.

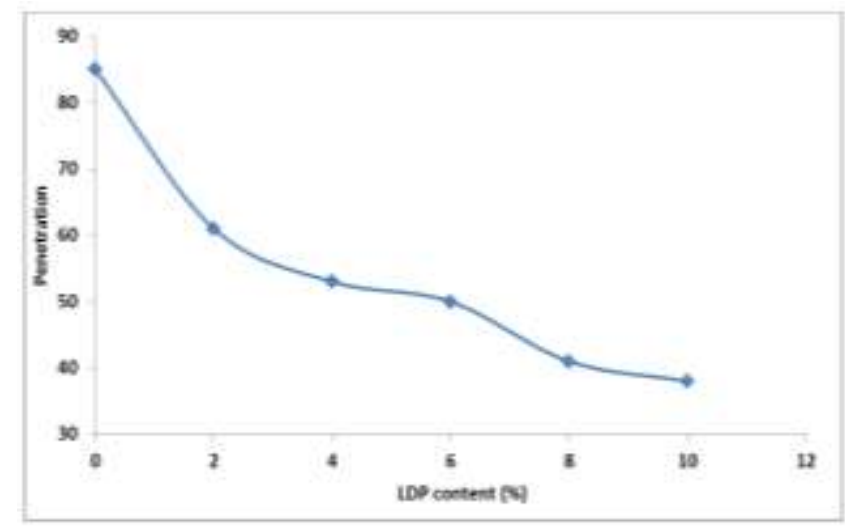

Figure 6: Penetration values of modified HMA

\subsection{Curve Fitting the Properties of modified Asphalt}

A polynomial curve with equation of the form of Equation 2 was assumed for stability values while a linear curve with equation of the form of Equation 3 was assumed for specific gravity, flow, softening point and penetration values.

$$
\begin{gathered}
y_{1}=\alpha_{2} X^{2}+\alpha_{1} X+\alpha_{0} \\
y_{2}=z_{1} X+z_{0}
\end{gathered}
$$

Where $\alpha_{2}, \alpha_{1}, \alpha_{0}=$ coefficient of polynomial Equation; $y_{1}=$ predicted stability values; $z_{1}, z_{0}=$ coefficient of linear Equation; $y_{2}=$ predicted asphalt property (flow, softening point and penetration values).

From Equation (2) and given $\mathrm{n}$ set of measurements, the least square estimates can be obtained. The sum of squared deviations of the experimental and predicted value is given as shown by Equation (4).

$$
S=\left(\sum y_{1}-\alpha_{2} X^{2}-\alpha_{1} X-\alpha_{0}\right)^{2}
$$

Minimizing and setting the partial derivative of the above Equation with respect to $\alpha_{2}, \alpha_{1}$ and $\alpha_{0}$ equal to zero, Equation (5) - (7) were developed.

$$
\begin{gathered}
\sum y_{1}=\alpha_{2} \sum X^{2}+\alpha_{1} \sum X+\alpha_{0} n \\
\sum X y_{1}=\alpha_{2} \sum X^{3}+\alpha_{1} \sum X^{2}+\alpha_{0} \sum X \\
\sum X^{2} y_{1}=\alpha_{2} \sum X^{4}+\alpha_{1} \sum X^{3}+\alpha_{0} \sum X^{2}
\end{gathered}
$$

Following the same algorithm, Equations (8) and (9) were developed from Equation (3)

$$
\begin{array}{r}
\sum y_{2}=z_{1} \sum X+z_{0} n \\
\sum X y_{2}=z_{1} \sum X^{2}+z_{0} \sum X
\end{array}
$$

\subsubsection{Coefficient of Stability Curve for Modified Asphalt Concrete.}

In determination of model coefficient for stability model Table 8 was developed.

By substituting into Equations (5) - (7), Equations (10)-(12) were obtained

$$
\begin{gathered}
40890=220 \alpha_{2}+300 \alpha_{1}+5 \alpha_{0} \\
245990=1800 \alpha_{2}+220 \alpha_{1}+30 \alpha_{0} \\
1804180=15664 \alpha_{2}+1800 \alpha_{1}+220 \alpha_{0}
\end{gathered}
$$

Solving simultaneously gives;

$\alpha_{2}=-12.41 ; \alpha_{1}=165.18 ; \alpha_{0}=7733$

Thus the second degree polynomial stability model with a $R^{2}$ value of $88.6 \%$ was deduced as shown by Equation (13).

$$
y_{1}=7733+165.18 x-12.41 x^{2}
$$


Table 8 Determining the Stability Model Coefficients

\begin{tabular}{lllllll}
\hline $\mathbf{X}$ & \multicolumn{1}{c}{$\boldsymbol{y}_{\mathbf{1}}$} & \multicolumn{1}{c}{$\boldsymbol{X}^{\mathbf{2}}$} & \multicolumn{1}{c}{$\boldsymbol{X}^{\mathbf{3}}$} & $\boldsymbol{X}^{\mathbf{4}}$ & $\mathbf{X} \boldsymbol{y}_{\mathbf{1}}$ & $\boldsymbol{X}^{\mathbf{2}} \mathbf{y}$ \\
2 & 8025 & 4 & 8 & 16 & 16050 & 32100 \\
4 & 8185 & 16 & 64 & 256 & 32740 & 130960 \\
6 & 8240 & 36 & 216 & 1296 & 49440 & 296640 \\
8 & 8340 & 64 & 518 & 4096 & 66560 & 532480 \\
10 & 8120 & 100 & 1000 & 10000 & 81200 & 812000 \\
$\sum X=$ & $\sum y_{1}=$ & $\sum X^{2}=$ & $\sum X^{3}=$ & $\sum X^{4}=$ & $\sum X y_{1}=$ & $\sum X^{2} y_{2}=$ \\
30 & 40890 & 220 & 1800 & 15664 & 245990 & 245990 \\
\hline
\end{tabular}

Table 10: Determining the Flow Model Coefficients

\begin{tabular}{rrrcc}
\hline $\mathrm{X}$ & $\mathrm{Y}_{2}$ & $\mathrm{X}^{2}$ & $\mathrm{XY}$ \\
\hline 2 & 3.10 & 4 & 6.2 \\
4 & 3.10 & 16 & 12.4 \\
6 & 3.0 & 36 & 18 \\
8 & 3.0 & 64 & 24 \\
& 2.9 & 100 & 29 \\
$\mathrm{XX}=30$ & & $\sum y_{2}=15.1$ & $\sum X^{2}=220$ & $\sum X y_{2}=89.6$
\end{tabular}

\subsubsection{Coefficient of Flow and other Properties and $R^{2}$ Value for Modified Asphalt}

a. Flow Model

Adopting Equation (8) and (9), the model coefficients for the flow model was developed using Table 10.

By substituting into Equations (8) and (9), Equations

(14) and (15) were obtained;

$$
\begin{array}{r}
15.1=30 Z_{1}+5 Z_{0} \\
89.6=220 z_{1}+30 z_{0}
\end{array}
$$

Solving simultaneously yields;

$$
z_{1}=0.025 ;=z_{0}=3.17
$$

Thus; the linear flow curve equation with a $R^{2}$ value of $89.2 \%$ becomes

$$
Y_{2}=3.17-0.025 \mathrm{x}
$$

Stepping through the same algorithm as above; the softening point, and penetration curves with their $R^{2}$ value can be obtained.

\section{b. Softening Point Curve}

The model obtained for softening point of modified asphalt is expressed by Equation (17) with a corresponding $\mathrm{R}^{2}$ value of $96.8 \%$.

$$
Y_{2}=52.4+1.1 \mathrm{x}
$$

\section{c. Penetration Curve}

The model obtained for penetration of modified asphalt is expressed in Equation (18) with a $\mathrm{R}^{2}$ value of $97.4 \%$

$$
Y_{2}=66-2.9 \mathrm{x}
$$

\subsection{Discussion of Result}

The modified asphalt concrete as observed from Figure 3a were higher than the conventional asphalt concrete mixtures. The increase in stability value can be attributed to increase in cohesion and adhesion properties caused by modifier introduction thereby causing internal friction. This improvement would enhance and improve fatigue resistance, thermal stress cracking and reduce temperature susceptibility and rutting occurrences as observed by [10]. Although, above $8 \%$ modifier addition, the reverse becomes the case. The flow values as observed from Figure $3 b$ decreased with LDP content. This may be inferred from the reduction in viscosity of binder with increase in modifier content.

The specific gravity values were noticed to increase with LDP content (Figure 4). The increase was drastic on introduction of LDP (0-2\%) after which, the increase became steady. The increase may be attributed to the difference in densities between the two substances.

The softening point values were noticed to increase with increase in addition of modifier. This may be attributed to increase in adhesive properties of modified bitumen as LDP content in creased. This is as depicted in Figure 5.

The penetration values of modified asphalt decreased with LDP content as depicted in Figure 6 
The models developed for the different properties of modified asphalt concrete collaborated well enough with the experimental values as was depicted and validated by the coefficient of determination values. The stability model is a polynomial model with $\mathrm{R}^{2}$ value of $88.69 \%$. The other models are linear models $\mathrm{R}^{2}$ value of $89.2 \%, 96.8 \%$ and $97.4 \%$ for the flow, softening point and penetration model respectively.

\section{CONCLUSION}

From the results and discussions of the study, the following conclusions are hereby made;

1. The stability of modified asphalt using L.D.P as bitumen modifier increased with LD content up to $8 \%$ after which, a decline was observed.

2. The softening point of modified asphalt increases with increased L.D.P content.

3. The flow and penetration values of modified asphalt decreases with increased L.D.P content.

4. The optimum replacement value of bitumen with L.D.P was found to be $8 \%$. Because the study is for medium traffic roads, further studies should be done considering heavy traffic roads.

\section{REFERENCES}

[1] Anonymous Asphalt concrete https://en.m.wikipedia.org/wiki/asphalt concret e, 2017.

[2] Christiana, F. A., Sandoval J. A., Jerez, A., Navaro, F. J., Martinez, F. J., Portal, P., Gallegos, C.. Evaluation of Thermal and Mechanical Properties of Recycled Polyethylene
Modified Bitumen. Polymer Testing; 27(8): 1005 - 1012. 2008.

[3] Isaacson, U., and Lu, X. Testing and Appraisal of Polymer Modified Road Bitumen. State of Art Materials and Structures, 28:139. 1997.

[4] Yildrim, Y. Polymer. Modified Asphalt Binders Contraction and Building Materials, 21(2): 6672, 2005.

[5] Mustafa, M. S. and Sufian Z.. "Prospects of Rubberised Bitumen Pavement for Malaysia Roads and Highways". Proceedings of the conference on the use of Rubberised Bitumen in Road Construction. 1997.

[6] Emesiobi, C. F., Bitumen and Tar. Testing and Quality Control of Materials in Civil and Highway Engineering. Port Harcourt Nigeria, Blue Print Limited, 2005.

[7] A \& C Plastics Inc. Types of plastics in the world. https://www.acplasticsinc.com/differenttypes-of-plastic-and-how-they-are-used. 2018.

[8] Desta, M. and Gebrexidan, A. The Environment Impacts of the Disposal of Plastic Rags and Water Bottles in Tigray, Northern Ethiopia Sacha Journal of Environmental Studies. Vol. 2(1). pp 81-94, 2012.

[9] ASTM D6926. Preparation of Bituminous Specimens Using Marshall Apparatus. 1992.

[10] Gonzaleez, U. O. "Rheological Property of Bitumen Modified with Polyethylene and Polyethylene Based Blends". Ph.D Thesis, University of Malasia, Kwala Lumpur, Malaysia. 2008. 